\title{
The importance of Mie resonances in ultra-black dragonfish skin pigment particles
}

\author{
Ishaan Lohia $\cdot$ Samuel Ahearn · Joseph Franjieh • \\ Marcel Di Vece
}

Received: 7 April 2021 / Accepted: 18 June 2021 / Published online: 3 July 2021

(C) The Author(s) 2021

\begin{abstract}
The ultra-black skin of the deep-sea dragonfish consists of small pigment particles which together provide optimal light absorption to prevent detection from bioluminescent predators or prey. The mechanism of light absorption in these pigment particles resembles the nanophotonic approaches to increase solar cell efficiency via Mie scattering and resonances. In this work, the Mie resonance responses of dragonfish pigment particles were investigated with finite-difference time-domain (FDTD) simulations to elucidate the exact mechanism responsible for the ultra-black skin of the dragonfish. Ellipsoidal pigment particles were found to have superior light absorption over spherical pigment particles. The pigment particles were also shown to exhibit forward scattering, demonstrating an important feature for repeated light absorption in pigment-containing skin layers. Although this work contributes to a deeper understanding of the ultra-back skin of the dragonfish, the nanophotonic mechanisms proposed here are likely more general, and could be applied to photovoltaic light management designs and immunometric detection based on light extinction.
\end{abstract}

I. Lohia $\cdot$ S. Ahearn $\cdot$ J. Franjieh $\cdot$ M. Di Vece $(\bowtie)$ Interdisciplinary Centre for Nanostructured Materials and Interfaces (CIMaINa) and Physics Department "Aldo Pontremoli", University of Milan, Via Celoria 16, 20133 Milan, Italy

e-mail: marcel.divece@unimi.it
Keywords Mie scattering - Dragon fish · Pigment particle $\cdot$ Camouflage $\cdot$ Deep-sea fish $\cdot$ Nanophotonics

\section{Introduction}

The dragonfish (Idiacanthus antrostomus) lives in the deep sea and is extremely light-absorbent-a camouflage tactic used to avoid being detected via bioluminescence from predators or prey. Its ultra-black skin was recently investigated by Davis et al. (2020) and was found to consist of assemblies of spherical and ellipsoidal pigment particles inside laminar layers (Davis 2020). The embedding skin medium has a refractive index which is identical to that of sea water to avoid a refractive index boundary and thereby prevent optical reflections. In general, the pigments, or melanosomes, of deep-sea fish have sizes between about 400 and $800 \mathrm{~nm}$ and a prevalent aspect ratio between about 1.2 and 3, making them mostly ellipsoidal (Riley 1997). Interestingly, the orientation of the pigments in the dragonfish skin is random (i.e., all orientations were present at each depth within the different layers of the skin).

Strong light absorption is also very important for solar cells, in which recent nanophotonic approaches have been developed to enhance light absorption, particularly in thin-film solar cells (Di Vece 2019; Giannakoudakis and Di Vece 2017). One promising technique is the use of Mie scatterers, which are particles or nanostructures with high refractive indices 
in which light can be captured and leaked into a solar cell (Brongersma et al. 2014; Neder et al. 2017). Mie resonances have been observed in light scattering and absorption by small particles whose size is on the order of the wavelength of incident light or larger (Mie 1908; Kerker 1969). Mie scattering increases the optical path length of incident light, leading to a higher optical absorption probability. Moreover, Mie light scattering is oriented mainly forward, directing light towards the photovoltaic region of interest (Wray and Atwater 2020; Tzarouchis and Sihvola 2018). A two-dimensional periodic array of subwavelength silicon nanocylinders designed to possess strongly substrate-coupled Mie resonances was shown to yield almost zero total reflectance over the entire spectral range from the ultraviolet to the near-infrared (Spinelli et al. 2012; Spinelli and Polman 2014). The strong and diverse interactions of Mie nanostructures with light were illustrated by the all-dielectric color printing of paintings by means of simple nanostructures fabricated with monocrystalline silicon, in which various brilliant color reflections were achieved by tuning the physical dimensions of the nanostructures (Nagasaki et al. 2017). Further applications for Mie scattering have been explored, such as using it in photocatalytic processes or combining with van der Waals materials (Xu 2012; Cihan 2018).

Although the work of Davis et al. simulated the strong optical absorption of dragonfish pigment particle assemblies via finite-difference time-domain (FDTD) methods, a detailed investigation into the interaction of light with the individual pigment particles remained unexplored (Davis 2020). Because the size of the dragonfish pigment particles allows for strong Mie-like properties, this work investigates the interaction of light with single-pigment particles via FDTD simulations to provide a deeper insight into the mechanisms of strong optical absorption in the dragonfish skin. Besides providing an interesting strategy for light management in photovoltaics, this work could also be applicable to immunometric detection based on light extinction and fluorescence quenching with latex or metal particles (Coletta and Amendola 2021; António et al. 2018; Bravin and Amendola 2020; Zhang 2019).

\section{Methods}

FDTD simulations, which solve the time-resolved Maxwell equations in three dimensions, were conducted using commercial software (Lumerical Solutions Inc.) to calculate the optical absorption in the pigment particles. A single dragonfish pigment particle was illuminated by a total-field scattered-field (TFSF) plane wave source, centrally incident along the z-axis. A background refractive index of 1.33 was used, equal to that of the dragonfish skin and seawater. The refractive indices of the pigment particles and the background tissue were obtained from the work of Davis et al. (2020). The simulations used a $1000 \times 1000 \times 100-\mathrm{nm}^{3}$, $2000 \times 2000 \times 2000-\mathrm{nm}^{3}$, and $3000 \times 3000 \times 3000$ $\mathrm{nm}^{3}$ simulation boxes for the $150-\mathrm{nm}$ and $450-\mathrm{nm}$ ellipsoidal and 450-nm spherical pigment particles, respectively, with perfectly matched layer (PML) boundaries of 12 layers. Data was collected for 50 linearly spaced frequency points. The mesh box size on and around the particle was varied between $10 \times 10 \times 10 \mathrm{~nm}^{3}$ and $15 \times 15 \times 15 \mathrm{~nm}^{3}$, depending on the size of the pigment particle, and was determined to be sufficiently accurate by convergence testing.

The size distribution of the dragonfish pigment particles, obtained by Davis et al. (2020), follows a mostly Gaussian distribution with particle diameter ranging between 600 and $800 \mathrm{~nm}$ (Davis 2020). Davis et al. (2020) also reports that particle aspect ratio ranges between 1 and 3 with a main Gaussian peak around 2 followed by a small peak at 2.5 . In accordance with these observations, this work simulated three ellipsoidal particles of 150-, 300-, and 450-nm radius (long axis) with an aspect ratio of 1.5. Only the 150- and 450-nm particles are discussed here because the $300-\mathrm{nm}$ particle fully supported the observed size-dependent trends. These particle diameters and aspect ratio were chosen to cover the mean and extreme particle sizes reported by Davis et al. (2020). The results shown in this work are from simulations in which the electric field of incident light was polarized in the direction of the $\mathrm{x}$-axis. The perpendicular (y-axis) polarization of incident light was also simulated and yielded slightly different results; however, the conclusions remained the same as for the $\mathrm{x}$-axis polarization. The optical absorption was calculated with respect to the effective surface area of the pigment particle from the reference of the incident light, i.e., the surface area in the plane of the light source (xy-plane). Optical absorption values greater than one were 
caused by the fact that the electromagnetic waves can also be absorbed from the sides of the pigment particle, due to the wave nature of light.

\section{Results and discussion}

To obtain insight into the pathway of light inside the pigment particles, the cross sections of the optical absorption, the electric field intensity, and the magnetic field intensity were calculated by integrating over the entire time of the simulation. In Fig. 1, the cross sections of the $150-\mathrm{nm}$ radius particle under incident light of 300-nm wavelength exhibit a very clear focal point at the bottom of the particle. This focal point resulted in increased absorption within the particle and a strong electric field going outward from the bottom. This strong forward scattering is
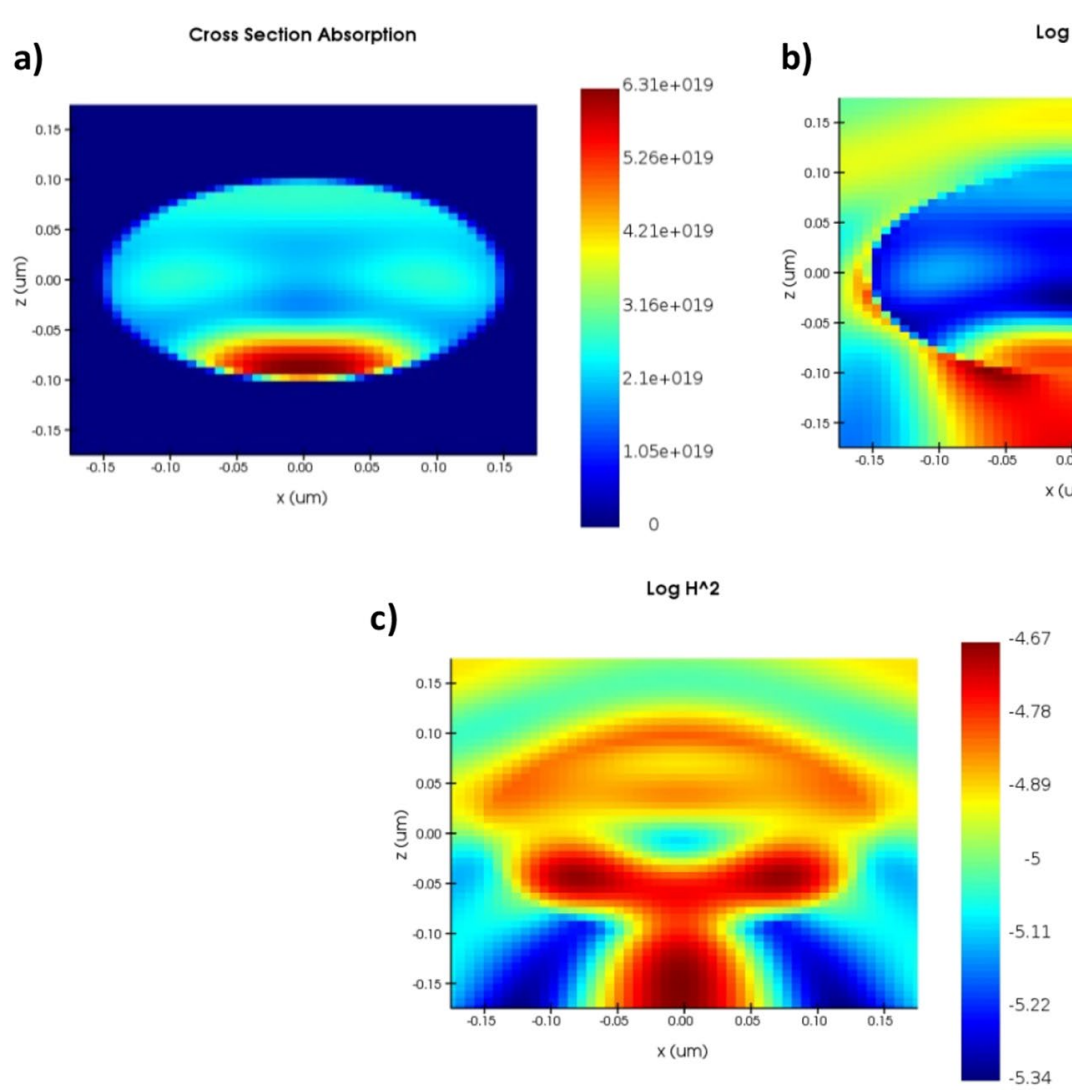

Fig. 1 Cross sections at $300 \mathrm{~nm}$ (wavelength) in xz-plane through the middle of the pigment particle with a long axis radius of $150 \mathrm{~nm}$ for a optical absorption, $\mathbf{b}$ integrated electric

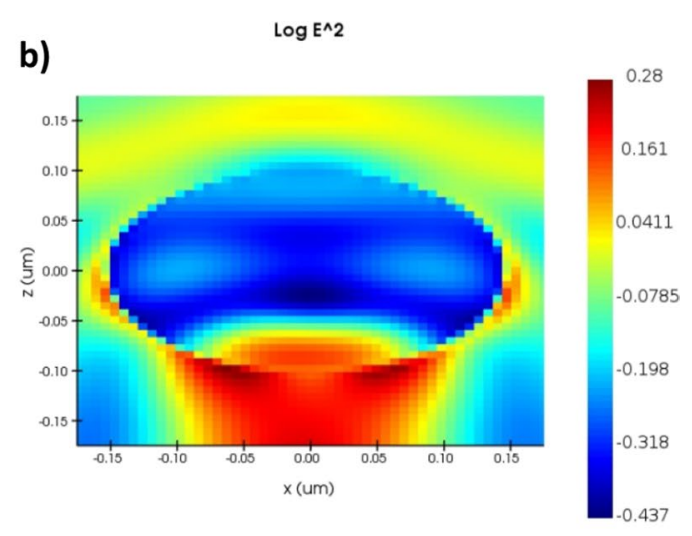

indicative of Mie-like behavior and is well-known from Mie theory (as derived from a series approximations to a solution for Maxwell's equations) (Kerker 1969) .

Evidence of light circulation within the pigment particle is provided by the observed regular patterns of the optical absorption and electric field intensity. The high-intensity areas indicate constructive wave interference while darker areas are caused by destructive interference. This interference pattern is only possible if part of the light was reflected within the particle. The less intense optical absorption and electric field intensity in the center of the particle is suggestive of light circulation around and below the surface of the particle, known as "whispering gallery modes." The cross section of the magnetic field intensity provides evidence of displacement current loops because the magnetic field profile is distinctly different from

field $\left(\mathrm{E}^{2}\right)$, and $\mathbf{c}$ integrated magnetic field $\left(\mathrm{H}^{2}\right)$. The high intensity of the optical absorption, $\mathrm{E}^{2}$, and $\mathrm{H}^{2}$ below the pigment particle suggest strong forward scattering 
that of the electric field intensity (Groep and Polman 2013). These dielectric displacement current loops further suggest that the light wave is circulating at different locations within the pigment particle.

The light interference patterns in the larger pigment particle with radius $450 \mathrm{~nm}$ had more maxima and minima for the 300-nm wavelength light than the $150-\mathrm{nm}$ radius particle, as shown in Fig. 2. From the optical absorption, electric field intensity, and magnetic field intensity, it is evident that most of the light was located under the surface of the pigment particle while the interior remained empty of electromagnetic energy. This again suggests a circulation of light underneath the pigment particle surface, likely the result of strong destructive interference in the center of the pigment particle. The low intensity of the electric field below the 450-nm pigment particle in Fig. 2c for $300 \mathrm{~nm}$ light was caused by strong
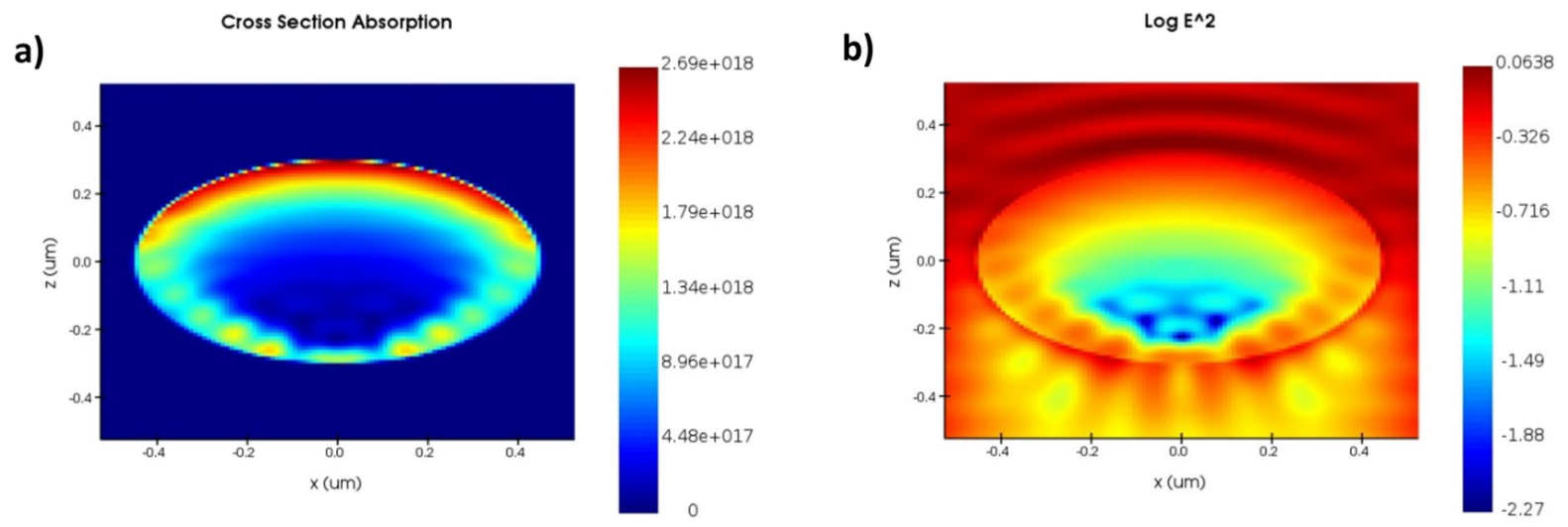

absorption in the pigment particle, leaving little light to be transmitted or scattered. The relatively intense electric field above this $450-\mathrm{nm}$ particle at a wavelength of $300 \mathrm{~nm}$ had about the same value as the $150-\mathrm{nm}$ pigment particle at a wavelength of $300 \mathrm{~nm}$, which means that there was no increase of reflection or backward scattering due to the particle size. This result demonstrates that not all pigment particles are optimized for forward scattering, which explains the rather large distribution of size and aspect ratio's in the natural occurrence of these pigment particles in the dragonfish skin. By providing a wide range of sizes and aspect ratio's, the dragonfish complements particles which exhibit strong absorption at particular wavelengths (larger particles) with particles that have strong forward scattering (smaller particles), resulting in an overall minimal reflection. Moreover, the fact that the $450-\mathrm{nm}$ radius pigment particles absorbed so

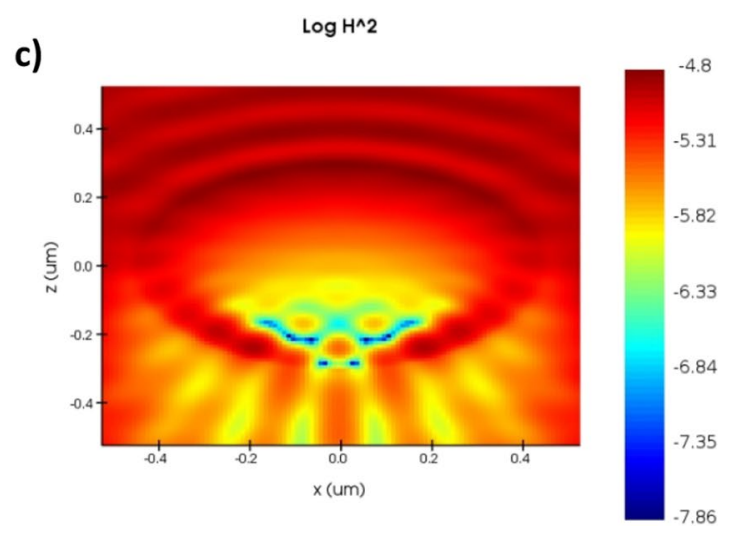

Fig. 2 Cross sections at $300 \mathrm{~nm}$ (wavelength) in xz-plane through the middle of the pigment particle with a long axis radius of $450 \mathrm{~nm}$ for a optical absorption, $\mathbf{b}$ integrated electric 
much light that Mie resonances are minimized could explain the upper size boundary found in pigment particles in biological samples. In agreement with the nature of optical interference, the cross sections of the larger pigment particle with radius of $450 \mathrm{~nm}$ at a wavelength of $658 \mathrm{~nm}$ (Fig. 3) were very similar to the smaller pigment particle (150-nm radius) at 300-nm wavelength (Fig. 1), because the interference pattern depends on the ratio of the particle size to the light wavelength.

The spectra of the optical absorption in the ellipsoidal pigment particles are shown in Fig. 4 as a function of light polarization and rotation angle of the particle. As stated previously, the optical absorption values greater than one were caused by the absorbance of light from the sides of the pigment particle due to the wave nature of light. As a result of the optical properties of the pigment molecule, the optical absorption

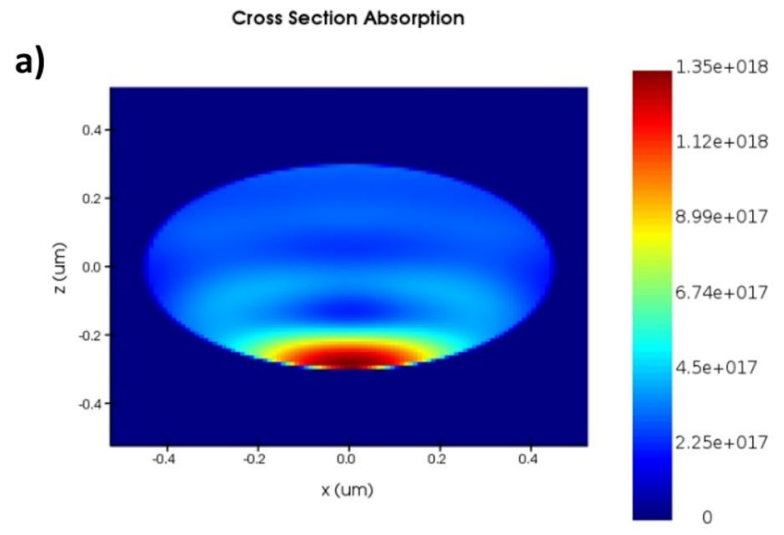

was strongest at small wavelengths and reduced considerably at larger wavelengths. For the 150-nm ellipsoidal pigment particle, greater rotation in the xz-plane increased overall optical absorption considerably because light passed through a longer pigment path, enhancing the absorption probability (i.e., less light was transmitted). The increase of light absorption upon rotation was particularly strong at shorter wavelengths, indicating that the optical resonances had a strong effect. Despite changes in intensity, the optical absorption curve profiles for the $150-\mathrm{nm}$ particle did not change significantly with rotation. The rotations for the 450-nm particle induced significant changes in both the optical absorption values and curve profile. Increasing the rotation angle mostly increased the optical absorption intensity and greater rotation angles corresponded to redshifted curve profiles. The 450-nm pigment particle exhibited optical absorption maxima

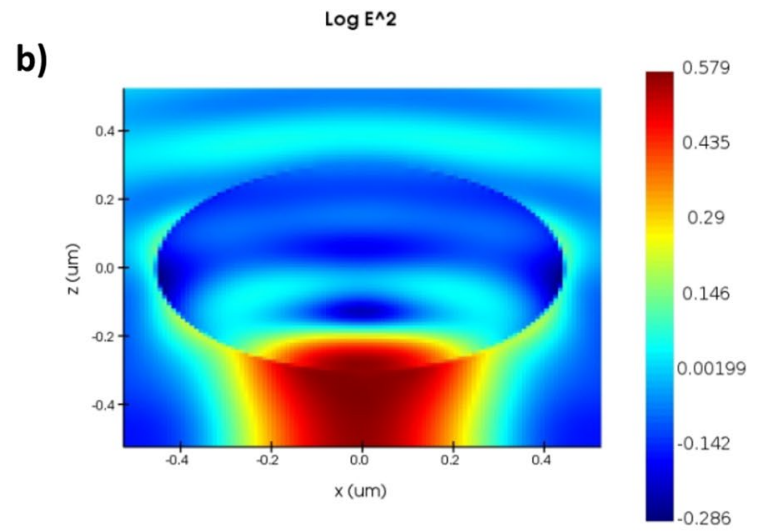

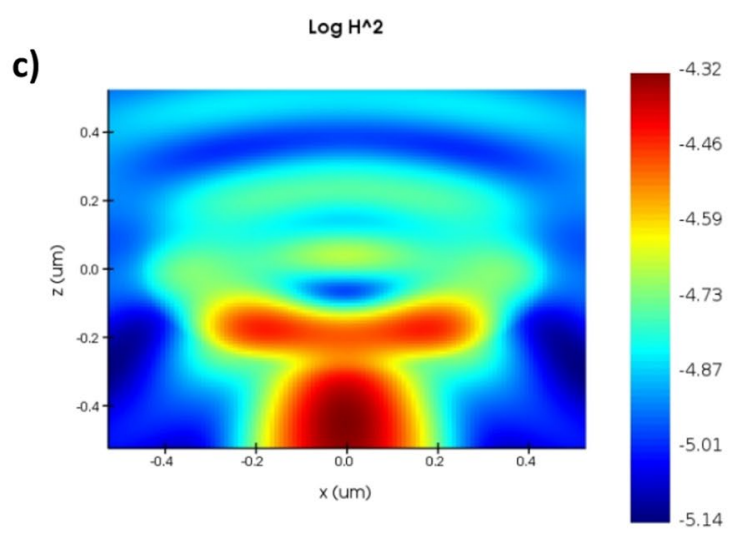

Fig. 3 Cross sections at $658 \mathrm{~nm}$ (wavelength) in xz-plane through the middle of the pigment particle with a long axis radius of $450 \mathrm{~nm}$ for a optical absorption, $\mathbf{b}$ integrated electric field $\left(\mathrm{E}^{2}\right)$, and $\mathbf{c}$ integrated magnetic field $\left(\mathrm{H}^{2}\right)$. The high inten- sity of the optical absorption, $\mathrm{E}^{2}<$ and $\mathrm{H}^{2}$ below the pigment particle suggest strong forward scattering. In agreement with Fig. 1 


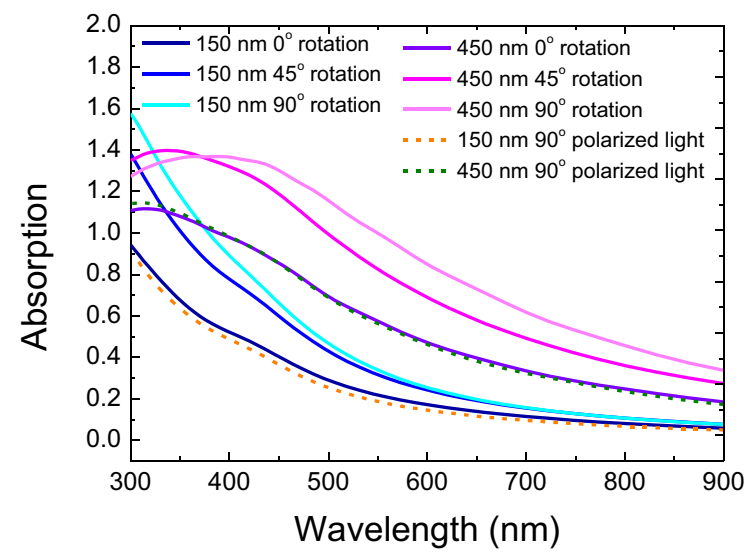

Fig. 4 Optical absorption spectra of ellipsoidal pigment particles of two sizes, under three rotations in the xz-plane and two opposite polarizations. The light is incident from the xy-plane normal to the z-direction, pointing downwards. For the 450-nm particle, the rotation has considerable effect on the absorption spectrum due to the presence of Mie resonances

at 320-, 338-, and 390-nm wavelengths for the $0^{\circ}, 45^{\circ}$, and $90^{\circ}$ rotations, respectively. Remarkably, the optical absorption at $90^{\circ}$ rotation around wavelengths of $300 \mathrm{~nm}$ was slightly reduced compared to at $45^{\circ}$ rotation, likely because of the presence of Mie resonances at $90^{\circ}$ rotation which were rather destructive. In contrast, the optical absorption in the $45^{\circ}$ rotated $150-\mathrm{nm}$ particle around wavelengths of $300 \mathrm{~nm}$ had increased due to Mie resonances. Although the rotation in the xz-plane of the 450-nm ellipsoidal particles had a significant effect, the differences were likely not enough to have them aligned in the most favorable position in the dragonfish skin. Alternatively, the slight differences in optical absorption between the different orientations of the ellipsoidal pigment particles may average out to an overall stronger optical absorption across the entire visible spectrum. The light polarization did not have a significant effect on the optical absorption of the ellipsoidal pigment particles. The optical absorption of the 450-nm pigment particle as compared with the 150$\mathrm{nm}$ pigment particle was much larger at longer wavelengths (3.2 times greater at $800 \mathrm{~nm}$ ) than at shorter wavelengths (1.6 times greater at $350 \mathrm{~nm}$ ). This is possibly due to Mie resonances which are present in the 450-nm particle but cannot be supported in the 150-nm particle at the longer wavelengths.

The z-component of the electric field in a spherical 450-nm particle, as shown in Fig. 5a, demonstrates the presence of interference below the pigment particle's a)
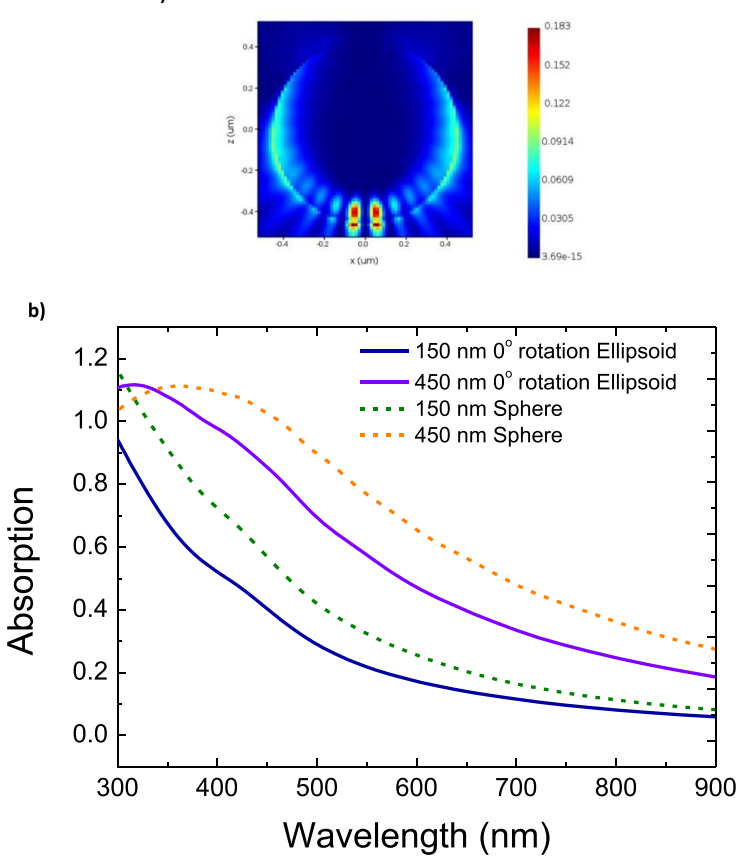

Fig. 5 a Cross section at $300 \mathrm{~nm}$ (wavelength) in xz-plane through the middle of the spherical pigment particle with radius of $450 \mathrm{~nm}$ of the $\mathrm{z}$-component of integrated the electric field. The constructive interference patterns are clearly visible and are indicative for light circulation within the particle. b Optical absorption spectra of ellipsoidal and spherical pigment particles. Although the optical absorption of the spherical particles appears larger than that of the ellipsoid particles, the total volume ratio between the spherical and ellipsoidal particles is 2.25 , resulting in less light absorption per pigment molecule than in the spherical particle

bottom surface and clearly illustrates light circulation. The z-component was chosen in this case because only light which has an electric field component in the z-direction (i.e., in a direction different than from the initial incidence) is shown. It appears that the high symmetry of the spherical particle favored this particular interference pattern. The spectra of the spherical pigment particles were compared with the spectra of the ellipsoidal particles in Fig. 5b and showed a remarkable difference. In the case of the $150-\mathrm{nm}$ pigment particle, the spherical particle absorbed significantly more light over the entire spectrum, especially at smaller wavelengths where the absorption was nearly 1.5 times greater. The same trend occurred for the $450-\mathrm{nm}$ particles with an additional red shift of the absorption maximum $(370 \mathrm{~nm})$. Considering that the total volume ratio between the spherical and ellipsoidal particles was 
2.25, the spherical particles absorbed less light per pigment molecule, which explains the presence of mostly ellipsoidal pigment particles in the dragonfish skin. It is clear from this comparison that the ellipsoidal pigment particles are optimal for light absorption per pigment molecule because they support more Mie resonances as consequence of their geometry. Interestingly, Mie resonances are only possible because the pigment molecule does not absorb light very strongly, leaving the possibility for multiple passages of the light wave.

\section{Conclusions}

Although a single-pigment particle absorbs only about 10 to $20 \%$ of incident light (depending on the wavelength), the forward scattering of Mie particles makes the subsequent layers of pigment particles very efficient, resulting in overall strong optical absorption across the visible light spectrum. Because of the presence of Mie resonances, which results in the circulation of light within the pigment particle that in turn increases the optical absorption probability, the incident light is absorbed over a much wider wavelength range than would be possible based solely on the optical absorption characteristics of the pigment molecules. It is tempting to conclude that nature selected the optimal size of pigment particles in the dragonfish skin to ensure the presence of Mie scattering in a trade-off between optical camouflaging benefits and other biological properties. Moreover, the ellipsoidal pigment particles were found to absorb much more light than the spherical pigment particles due to Mie resonances, which explains the presence of mostly ellipsoidal pigment particles in the skin of the dragonfish. Because pigment particles in other creatures have similar shapes and sizes, the presented analysis may be a general phenomenon. Moreover, the understanding of the dragonfish skin pigment particles provided in this work could be an inspiration for novel concept solar cell designs and offer insight for developing ultra-black materials.

Funding Open access funding provided by Università degli Studi di Milano within the CRUI-CARE Agreement.

\section{Declarations}

Conflict of interest The authors declare that they have no conflict of interest.

Open Access This article is licensed under a Creative Commons Attribution 4.0 International License, which permits use, sharing, adaptation, distribution and reproduction in any medium or format, as long as you give appropriate credit to the original author(s) and the source, provide a link to the Creative Commons licence, and indicate if changes were made. The images or other third party material in this article are included in the article's Creative Commons licence, unless indicated otherwise in a credit line to the material. If material is not included in the article's Creative Commons licence and your intended use is not permitted by statutory regulation or exceeds the permitted use, you will need to obtain permission directly from the copyright holder. To view a copy of this licence, visit http://creativecommons.org/licenses/by/4.0/.

\section{References}

António M et al (2018) Functionalized gold nanoparticles for the detection of C-reactive protein. Nanomaterials 8(4). https://doi.org/10.3390/nano8040200

Bravin C, Amendola V (2020) Wide range detection of C-Reactive protein with a homogeneous immunofluorimetric assay based on cooperative fluorescence quenching assisted by gold nanoparticles. Biosens Bioelectron 169:112591. https://doi.org/10.1016/j.bios.2020.112591

Brongersma ML, Cui Y, Fan S (2014) Light management for photovoltaics using high-index nanostructures. Nat Mater 13(5):451-460

Cihan AF et al (2018) Silicon Mie resonators for highly directional light emission from monolayer MoS2. Nat Photonics 12(5):284-290. https://doi.org/10.1038/s41566-018-0155-y

Coletta G, Amendola V (2021) Numerical modelling of the optical properties of plasmonic and latex nanoparticles to improve the detection limit of immuno-turbidimetric assays. Nanomaterials 11(5). https://doi.org/10.3390/nano11051147

Davis AL et al (2020) Ultra-black camouflage in deep-sea fishes. Curr Biol 30(17):3470-3476.e3. https://doi.org/10. 1016/j.cub.2020.06.044

Di Vece M (2019) Using nanoparticles as a bottom-up approach to increase solar cell efficiency. KONA Powder Part 36:72-87. https://doi.org/10.14356/kona.2019005

Giannakoudakis G, Di Vece M (2017) Improving optical absorption in a-Si thin films with $\mathrm{TiO} 2$ Mie scatterers. In European Physical Journal D 71(4), Springer, 233 Spring ST, New York, NY 10013 USA. https://doi.org/10.1140/ epjd/e2017-70570-7

Kerker M (1969) The scattering of light and other electromagnetic radiation, 1st edn. Academic Press, New York

Mie G (1908) Beiträgezur Optik trüber Medien, speziell kolloidaler Metallösungen. Annalen der Physik 330(3): 377445, John Wiley \& Sons, Ltd. https://doi.org/10.1002/ andp. 19083300302

Nagasaki Y, Suzuki M, Takahara J (2017) All-dielectric dualcolor pixel with subwavelength resolution. Nano Lett 
17(12): 7500-7506. American Chemical Society. https:// doi.org/10.1021/acs.nanolett.7b03421

Neder V, Luxembourg SL, Polman A (2017) Efficient colored silicon solar modules using integrated resonant dielectric nanoscatterers. Appl Phys Lett 111(7) 073902. American Institute of Physics. https://doi.org/10.1063/1. 4986796

Riley PA (1997) Melanin. Int J Biochem Cell Biol 29(11):12351239. https://doi.org/10.1016/S1357-2725(97)00013-7

Spinelli P, Polman A (2014) Light trapping in thin crystalline si solar cells using surface Mie scatterers. IEEE J Photovoltaics 4(2):554-559. https://doi.org/10.1109/JPHOTOV. 2013.2292744

Spinelli P, Verschuuren MA, Polman A (2012) Broadband omnidirectional antireflection coating based on subwavelength surface Mie resonators. Nat Commun 3:692

Tzarouchis D, Sihvola A (2018) Light scattering by a dielectric sphere: perspectives on the Mie resonances. In Applied Sciences-Basel 8(2), MDPI, ST ALBAN-ANLAGE 66, CH-4052 Basel, Switzerland. https://doi.org/10.3390/ app8020184

van de Groep J, Polman A (2013) Designing dielectric resonators on substrates: combining magnetic and electric resonances. Opt Express 21(22):26285-26302. https://doi. org/10.1364/OE.21.026285

Wray PR, Atwater HA (2020) Light-matter interactions in films of randomly distributed unidirectionally scattering dielectric nanoparticles. ACS Photonics 7(8): 2105-2114. American Chemical Society. https://doi.org/10.1021/acsphotonics.0c00545

$\mathrm{Xu} \mathrm{H}$ et al (2012) Size-dependent Mie's scattering effect on $\mathrm{TiO} 2$ spheres for the superior photoactivity of $\mathrm{H} 2$ evolution. J Phys Chem C 116(5):3833-3839. https://doi.org/ 10.1021/jp209378t

Zhang X et al (2019) Förster resonance energy transfer (FRET)-based biosensors for biological applications. Biosens Bioelectron 138:111314. https://doi.org/10.1016/j.bios.2019.05.019

Publisher's note Springer Nature remains neutral with regard to jurisdictional claims in published maps and institutional affiliations. 